\title{
INDIRECT EXCHANGE IN BAND-INVERTED HETEROJUNCTIONS OF IV-VI SEMIMAGNETIC COMPOUNDS
}

\author{
V.K. Dugaev, V.I. Litvinov \\ Chernovtsy Department of the Institute of Materials Science Problems \\ Ukrainian Academy of Sciences \\ I. Wilde 5, 274001 Chernovtsy, Ukraine
}

AND M. OsZWAEDowsKI

Technical University of Poznań, Piotrowo 3, 60-965 Poznań, Poland

The interaction of spins localized within the band-inverted heterojunction with the Weyl excitations is shown to differ significantly from the usual $s-d$ interaction. The indirect exchange interaction is long-range and antiferromagnetic. The magnitude of interaction decreases with the distance as $R^{-3}$. The effective interaction depends on the parameters of electron energy spectrum and parameters of the heterojunction as well. It consists of anisotropic Heisenberg term and of pseudodipole terms.

PACS numbers: 73.20.Dx, 75.30.Et, 75.30.Hx

The investigation of indirect exchange interaction in semiconductors is of great importance because of the problem of determination of magnetic phase structure of semimagnetic semiconductors at low temperatures.

The formulas of effective interaction are known for three-dimensional crystals such as metals (RKKY), direct-gap [1], indirect-gap [2], small-gap and zero-gap [3-5] semiconductors. The indirect interaction is responsible for the existence of ferromagnetic and spin-glass states in metals and IV-VI semimagnetic semiconductors [6-8].

In the present paper we examine the interaction of magnetic atoms in inverted heterojunction, i.e. in such a case when heterojunction is formed of small-gap semiconductors with mutually inverted bands (for instance, $\mathrm{PbTe} / \mathrm{Pb}_{1-x} \mathrm{Sn}_{x} \mathrm{Te}$ with $x>0.36$ ). It was shown in [9-11] that in this case the carriers are localized in the neighborhood of the interface, and the energy spectrum of these excitations is gapless, $E(k)= \pm v k$. This property of the spectrum is of crucial importance because, as it follows from the general reasons, the interaction of magnetic impurities via zero-gap electron excitations should be long-range. In order to make the 
details of this mechanism clear the calculations are required taking into account particularities of the interband and spin-orbit couplings, which are characteristic of the energy spectrum of IV-VI semiconductors.

The initial Hamiltonian of electrons belonging to one of $L$-valleys is written in the basis of wave functions $L_{6}^{ \pm}, L_{6}^{ \pm}$[12]:

$$
H_{0}=\left(\begin{array}{cccc}
\Delta & 0 & -\mathrm{i} v k_{z} & \mathrm{i} v k_{-} \\
0 & \Delta & -\mathrm{i} v k_{+} & -\mathrm{i} v k_{z} \\
\mathrm{i} v k_{z} & \mathrm{i} v k_{-} & -\Delta & 0 \\
-\mathrm{i} v k_{+} & \mathrm{i} v k_{z} & 0 & -\Delta
\end{array}\right),
$$

where $|\Delta|$ is half-width of the gap, $v / h$ is the interband matrix element of the velocity operator, $k_{ \pm}=k_{x} \pm \mathrm{i} k_{y}, z$-axis is directed along the unit vector $n$ which points to a given $L$-extremum of the Brillouin zone. For simplicity the Hamiltonian is taken to be isotropic.

The choice of the basis suggests the following form of the $s-d$ coupling for an impurity spin $\boldsymbol{S}_{i}$ situated at $\boldsymbol{R}_{i}$ :

$$
H_{\text {int }}=\left(\begin{array}{cc}
g_{1} \sigma \cdot S_{i} & 0 \\
0 & g_{2} \sigma \cdot S_{i}
\end{array}\right) \delta\left(r-R_{i}\right),
$$

where $g_{1}$ and $g_{2}$ are the matrix elements of $s-d$ coupling.

We can transform (1) to the usual form of the Dirac Hamiltonian by unitary transformation $U=\operatorname{diag}(1,1, \mathrm{i},-\mathrm{i})$ :

$$
H_{0}=\Delta \tau_{z}+v \tau_{x} \boldsymbol{\sigma} \cdot \boldsymbol{k} .
$$

Correspondingly, instead of (2) we get

$$
\begin{aligned}
H_{\text {int }} & =\frac{1}{2}\left[\left(g_{1}-g_{2}\right) \boldsymbol{\sigma} \cdot S_{i}+\left(g_{1}+g_{2}\right) \tau_{z} \sigma \cdot S_{i}+2 g_{2}\left(1-\tau_{z}\right)(\sigma \cdot n)\left(S_{i} \cdot n\right)\right] \\
& \times \delta\left(\boldsymbol{r}-\boldsymbol{R}_{i}\right) .
\end{aligned}
$$

Let a junction be situated at $z=0$. The inverted heterojunction can be modeled [9] by considering $\Delta=\Delta(z)$ with $\operatorname{sgn}(\Delta(z))=-\operatorname{sgn}(\Delta(-z))$. For $k_{x}=$ $k_{y}=0$ the Hamiltonian (3) has two eigenfunctions corresponding to localized excitations

$$
\begin{aligned}
& \Psi_{1}=\frac{A}{\sqrt{2}}\left(\begin{array}{c}
1 \\
0 \\
\mathrm{i} \\
0
\end{array}\right) \exp \left(-\frac{1}{v} \int_{0}^{z} \Delta(z) \mathrm{d} z\right), \\
& \Psi_{2}=\frac{A}{\sqrt{2}}\left(\begin{array}{c}
0 \\
1 \\
0 \\
-\mathrm{i}
\end{array}\right) \exp \left(-\frac{1}{v} \int_{0}^{z} \Delta(z) \mathrm{d} z\right),
\end{aligned}
$$

where $A$ is for normalization.

Using (5) as a new basis for (3), we obtain

$$
H_{0}=v\left(-\sigma_{x} k_{y}+\sigma_{y} k_{x}\right)
$$


and

$$
H_{\mathrm{int}}=A^{2} \boldsymbol{\sigma} \cdot \boldsymbol{\Sigma} \delta\left(r-\boldsymbol{R}_{i}\right),
$$

where

$$
\Sigma_{x, y}=\frac{1}{2}\left(g_{1}+g_{2}\right) S_{x, y}-g_{2} n_{x, y}(n \cdot S), \quad \Sigma_{z}=\frac{1}{2}\left(g_{1}-g_{2}\right) S_{z}+g_{2} n_{z}(n \cdot S) .(8)
$$

The Hamiltonian (6) describes gapless Weyl particles with $E(k)= \pm v k$.

Let us consider the interaction of two magnetic impurities situated at the junction plane. In the loop approximation we have

$$
E_{\text {int }}(R)=-\mathrm{iSp} \int \frac{\mathrm{d} \varepsilon}{2 \pi} H_{\mathrm{int}, 1} G_{0}(\varepsilon, \boldsymbol{R}) H_{\mathrm{int}, 2}^{+} G_{0}(\varepsilon,-\boldsymbol{R}),
$$

where $G_{0}$ is the Green function of Hamiltonian (6) in $\varepsilon, \boldsymbol{R}$ representation.

Aftẹr calculating (9) we obtain

$$
\begin{aligned}
& E_{\text {int }}(R)=-\frac{A^{4}}{4 \pi^{2} v R^{3}}\left[\left(C_{0}+C_{1}\right) \Sigma_{1 z} \Sigma_{2 z}+\left(C_{0}-C_{1}\right) \Sigma_{1 \perp} \cdot \Sigma_{2 \perp}\right. \\
& \left.\quad+2 C_{1}\left(\Sigma_{1} \cdot \xi\right)\left(\Sigma_{2} \cdot \xi\right)\right]
\end{aligned}
$$

where $\xi=\boldsymbol{R} / R, C_{0}$ and $C_{1}$ are the following constants, which we calculated numerically

$$
\begin{aligned}
& C_{0}=\iint_{0}^{\infty} \frac{x_{1} x_{2}}{x_{1}+x_{2}} J_{0}\left(x_{1}\right) J_{0}\left(x_{2}\right) \mathrm{d} x_{1} \mathrm{~d} x_{2} \approx 0.172, \\
& C_{1}=\iint_{0}^{\infty} \frac{x_{1} x_{2}}{x_{1}+x_{2}} J_{1}\left(x_{1}\right) J_{1}\left(x_{2}\right) \mathrm{d} x_{1} \mathrm{~d} x_{2} \approx 0.578,
\end{aligned}
$$

and $J_{0}(x), J_{1}(x)$ are the Bessel functions.

The expression (10) takes into account only the contribution of one $L$-valley. The summation over all the valleys is performed by assuming that the $z=0$ plane lies in (111) crystallographic plane. We get finally

$$
\begin{aligned}
E_{\mathrm{int}} & \approx-0.019 \frac{A^{4} g_{1}^{2}}{v R^{3}}\left[\left(1+t^{2}-t\right) S_{1 z} S_{2 z}-0.542\left(1-0.0665 t^{2}+\frac{t}{2}\right) S_{1 \perp} \cdot S_{2 \perp}\right. \\
& \left.+1.542\left(1+\frac{t^{2}}{4}+\frac{t}{2}\right)\left(S_{1} \cdot \xi\right)\left(S_{2} \cdot \xi\right)\right],
\end{aligned}
$$

where $t=g_{2} / g_{1}$. Thus, the effective interaction consists of $X X Z$-Heisenberg terms and of a pseudodipole one (the last term in (13)). The interaction depends on the distance between the impurity spins as $R^{-3}$.

The energetically favorable magnetic ordering can be determined by comparing the energy gains for different mutual orientations of spins: (a) they are perpendicular to the junction plane, (b) they lie in the plane with $\boldsymbol{S}_{1}, \boldsymbol{S}_{2}$ being perpendicular to $\xi$, and (c) they are collinear with $\xi$. It appears that when $0<g_{2} / g_{1}<2.46$ the ferromagnetic ordering is energetically favorable with both spins directed along $\boldsymbol{\xi}$. In other cases the ground state corresponds to the ferromagnetically ordered pair of spins directed perpendicular to the junction plane. 


\section{References}

[1] N. Bloembergen, T. Rowland, Phys. Rev. 97, 1679 (1955).

[2] A.A. Abrikosov, J. Low Temp. Phys. 33, 505 (1978).

[3] L. Liu, G. Bastard, Phys. Rev. B 25, 487 (1982).

[4] G. Bastard, C. Leviner, Phys. Rev. B 20, 4256 (1979).

[5] V.K. Dugaev, V.I. Litvinov, Phys. Rev. B 41, 788 (1990).

[6] M. Escorne, A. Mauger, J.L. Tholence, R. Triboulet, Phys. Rev. B 29, 6306 (1984).

[7] T. Story, R.R. Galazzka, R.B. Frankel, P.A. Wolf, Phys. Rev. Lett. 56, 777 (1986).

[8] H.J.M. Swagten, H.v.d. Hoek, W.J.M. de Jonge, R.R. Gałazzka, P. Warmenbol, J.T. Devreese, in: Proc. 19th Int. Conf. on the Phys. of Semiconductors, PWN, Warszawa 1988, p. Fr-A-I.5.

[9] B.A. Volkov, O.A. Pankratov, Pisma Zh. Eksp. Teor. Fiz. 42, 145 (1985); 42, 178 (1985).

[10] D. Agassi, V. Korenman, Phys. Rev. B 37, 10095 (1988).

[11] V. Korenman, H.D. Drew, Phys. Rev. B 35, 6446 (1987).

[12] D.L. Mitchell, R.F. Wallis, Phys. Rev. 151, 581 (1966). 